\title{
FUNCTIONS WHOSE HIGHER POWERS ARE ORTHOGONAL TO A GIVEN FUNCTION ${ }^{1}$
}

\author{
BURTON RANDOL AND NEIL RICKERT
}

In this note, we shall establish the following result:

Theorem. Suppose $k$ is an integer $\geqq 0, a<b$, and $m(t) \not \equiv 0$ is a complex-valued function of class $C^{k}$ on $[a, b]$ (taking left-and right-handed derivatives at the endpoints). Then there exists a complex-valued function $F(t)$, of class $C^{k+1}$ on $[a, b]$, such that the following conditions are satisfied:

1.

$$
\begin{aligned}
\int_{a}^{b}(F(t))^{n} m(t) d t & =1 \text { if } n=1, \\
& =0 \text { if } n \text { is an integer }>1 .
\end{aligned}
$$

2.

$$
F^{(j)}(a)=F^{(j)}(b)=0 \quad(j=0, \cdots, k+1) .
$$

In our proof of this result, we may assume that $m(t) \neq 0$ for $t$ in $[a, b]$, and that $C \neq 0$, where $C$ is the integral of $m(t)$ over $[a, b]$. To see this, note that $[a, b]$ always possesses a subinterval $\left[a^{\prime}, b^{\prime}\right]$, over which both $m(t)$ and its integral are nonzero. A solution to the problem can thus be obtained by first solving the corresponding problem for $\left[a^{\prime}, b^{\prime}\right]$, and then extending the resulting function to $[a, b]$ by defining it to be zero outside $\left[a^{\prime}, b^{\prime}\right]$. We may also assume, by a linear transformation if necessary, that $a=0$ and $b=2 \pi$. Finally, we may assume that $C=2 \pi$, for if this is not initially the case, we can bring it about by replacing $m(t)$ by $2 \pi m(t) / C$.

Definition. For $\eta$ in $(0,1)$, define $g_{\eta}(t)$ to be the function inverse to $t+\eta \sin t(0 \leqq t \leqq 2 \pi)$. Note that $g_{\eta}(0)=0$, and $g_{\eta}(2 \pi)=2 \pi$.

REMARK. It is evident by Rouché's theorem, that for sufficiently small $\eta, g_{\eta}(t)$ can be holomorphically extended into any fixed disc about the origin.

Definition. For $\eta$ in $(0,1)$, define $f_{\eta}(t)=\left(\exp i g_{\eta}(t)\right) / \pi \eta$. Note that $f_{\eta}(0)=f_{\eta}(2 \pi)=1 / \pi \eta$.

Lemma. For any $\eta$ in $(0,1)$,

$$
\begin{aligned}
\int_{0}^{2 \pi}\left(f_{\eta}(t)\right)^{n} d t & =1 \text { if } n=1, \\
& =0 \text { if } n \text { is an integer }>1 .
\end{aligned}
$$

Received by the editors May 5, 1966.

1 The authors were partially supported by the National Science Foundation, under Grants NSF-GP-1814, and NSF-GP-5493, respectively. 
Proof. Making the substitution $s=g_{\eta}(t)$, we see that

$$
\begin{aligned}
(\pi \eta)^{-n} \int_{0}^{2 \pi} e^{i n g_{\eta}(t)} d t & =(\pi \eta)^{-n} \int_{0}^{2 \pi} \frac{e^{i n}}{g_{\eta}^{\prime}(l)} d s \\
& =(\pi \eta)^{-n} \int_{0}^{2 \pi} e^{i n s}(1+\eta \cos s) d s,
\end{aligned}
$$

and the conclusion of the lemma follows immediately.

Proof of the Theorem. For $t$ in $[0,2 \pi]$, define $M(t)=\int_{0}^{t} m(s) d s$, and denote by $\Gamma$ the curve traced out in the complex plane by $M(t)$, as $t$ runs from 0 to $2 \pi$. Note that $\Gamma$ begins at 0 and terminates at $2 \pi$. Now let $\eta$ be so small that $f_{\eta}(t)$ is holomorphic in a disc about the origin which contains $\Gamma$. Then

$$
\begin{aligned}
\int_{0}^{2 \pi}\left(f_{\eta}(M(t))\right)^{n} m(t) d t & =\int_{\Gamma}\left(f_{\eta}(z)\right)^{n} d z \\
& =\int_{0}^{2 \pi}\left(f_{\eta}(t)\right)^{n} d t,
\end{aligned}
$$

where the last equality follows from Cauchy's theorem.

Thus, if we define $F(t)=f_{\eta}(M(t))\left(1-\pi \eta f_{\eta}(M(t))\right)^{k+2}$, it follows from (1), and from the fact that $f_{\eta}(0)=f_{\eta}(2 \pi)=1 / \pi \eta$, that we are done.

REMARKs. 1. By defining $g_{\eta}(t)$ to be the function inverse to

$$
t+\eta\left(\sum_{j=1}^{n} a_{j} \frac{\sin N_{j} t}{N_{j}}\right)
$$

we can arbitrarily prescribe the values of any finite number of the integrals in (1) of the theorem.

2. There are several questions which can be asked about possible relationships between the degrees of regularity of the functions $F(t)$ and $m(t)$ which occur in the theorem. For example, if $m(t)$ is $C^{\infty}$ with compact support in $[a, b]$, is there always an $F(t)$ in the same class which satisfies (1) of the theorem? Similarly, if $m(t)$ is holomorphic in a neighborhood of $[a, b]$, is there always an $F(t)$ in the same class which satisfies (1) of the theorem?

YALE UNIVERSITY 